\title{
Schlaganfall nach Wirbelsäulen-Manipulationen?
}

\section{Ein internationales Team von Chiro- praktikern ist in einem Review der Frage nachgegangen, ob Manipula- tionen der oberen Wirbelsäule einen Schlaganfall verursachen können.}

- Die Autoren trugen alle Fall-KontrollStudien zum Thema zusammen, beurteilten ihre methodische Qualität und unternahmen den Versuch, eine Synthese der Daten zu erstellen. Insgesamt fanden sich vier Untersuchungen, die den Einschlusskriterien entsprachen. Nur zwei davon waren von ausreichender Qualität. Eine dieser Studien sprach für und die andere gegen einen Kausalzusammenhang. Die Autoren folgern, dass verlässliche Evidenz weder für noch gegen die These existiert, dass zervikale Manipulationen einen Schlaganfall verursachen können.

\section{Kommentar}

Kaum ein Thema der Komplementärmedizin erregt die Gemüter mehr als dieses. Auf der einen Seite stehen Manualtherapeuten, die standhaft die Meinung vertreten, dass ihre Behandlungsformen harmlos sind. Auf der anderen Seite stehen Neurologen und andere Experten, die die Datenlage so einschätzen, dass zumindest Bedenken angebracht sind. Beide scheinen gute Argumente für ihre Thesen zu haben, sodass hier eigentlich nur aussagekräftige Forschung helfen kann. Leider führt die vorliegende Analyse nur wenig weiter, denn die publizierten FallKontroll-Studien ergeben eine fast klassische Patt-Situation. In dieser Lage wäre es hilfreich, auf andere Daten zurückzugreifen. Hätten die Autoren z. B. Fallberichte mit in Betracht gezogen, dann wäre das Bild wohl klarer geworden. Derzeit sind mehrere hundert Fälle publiziert, bei denen chiropa- raktische Manipulationen der oberen Wirbelsäule zu sehr ernsten Zwischenfällen mit z. T. permanenten neurologischen Defiziten oder sogar zum Tod geführt haben. Natürlich sind solche Berichte nichts weiter als Anekdoten mit wenig Gewicht, in der Gesamtschau ergeben sie jedoch durchaus wertvolle Hinweise. Wie immer die Diskussion um dieses Thema auch ausgehen mag, aus meiner Sicht steht bis zum Vorliegen wirklich überzeugender Daten eines fest: Therapeuten, die solche Therapien einsetzen, sollten ihre Patienten zumindest über das Risiko aufklären - und ich freue mich, dass auch die Autoren dieser Analyse diese Meinung deutlich vertreten. E. ERNST *

- M. J. Haynes et al.

Assessing the risk of stroke from neck manipulation: a systematic review. Int. J. Clin. Pract. 2012 Oct;66(10):940-7. doi: 10.1111/j.17421241.2012.03004.x.

\section{Mehr Klarheit beim zytologisch unklaren Schilddrüsenknoten}

\begin{abstract}
Mithilfe einer genetischen Expressionsanalyse gelingt eine bessere Einordnung zytologisch unklarer Punktate von Schilddrüsenknoten.
\end{abstract}

— Schilddrüsenknoten sind ein extrem häufiger Befund und meistens gutartig. Im diagnostischen Algorithmus spielt der zytologische Befund des Knotens eine entscheidende Rolle. Allerdings ist in 15-30\% der Feinnadelbiopsate mit einem zytologisch nicht einzuordnenden Befund zu rechnen.

Dieses Dilemma wurde nun in einer auf 19 Monate angelegten prospektiven multizentrischen Untersuchung an fast 4000 Patienten mit 4812 Feinnadelaspiraten von Knoten über $1 \mathrm{~cm}$ Durchmesser mithilfe eines neuen genetischen Tests teilweise gelöst. In 577 Fällen fanden sich zytologisch unklare Aspirate, die in 413 Fällen mit operativ gewon- nenen histopathologischen Präparaten derselben Region verglichen werden konnten. Unter Beachtung bestimmter Einschlusskriterien konnte bei 265 zytologisch unklaren Knoten ein molekularbiologischer Test zur Feststellung genetischer Mutationen angewandt werden.

Von diesen 265 Knoten mit unklarer Zytologie wiesen 85 einen malignen histopathologischen Befund auf. Mithilfe des molekularbiologischen Tests gelang es, 78 dieser 85 malignen Knoten als solche zu klassifizieren. Sieben Aspirate mit falsch negativen Ergebnissen erwiesen sich als so zellarm, dass sie wegen ungenügender Punktionstechnik als nicht untersuchbar eingeordnet werden mussten.

\footnotetext{
- E.K. Alexander et al. Preoperative diagnosis of benign thyroid nodules with indeterminate cytology. New Engl. J. Med. 2012; 367: 705-715
}

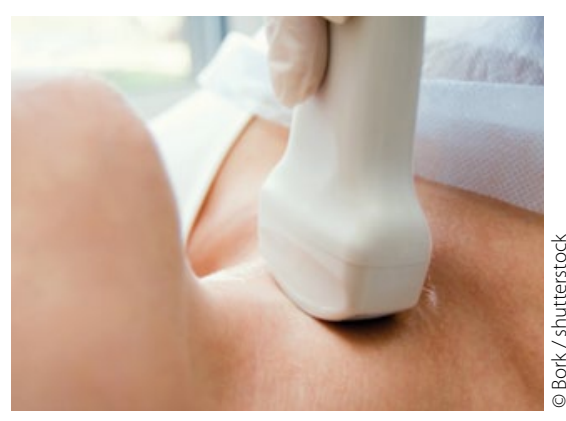

Ist der Konten gutartig oder nicht? Da hilft auch die Zytologie nicht immer.

\section{Kommentar}

Bei Schilddrüsenknoten ist jede Untersuchungsmethode willkommen, die mehr Klarheit ins Geschehen bringt und die Entscheidung zur Operation oder zum Zuwarten auf ein möglichst sicheres Fundament stellt. Trotz klarer Algorithmen und ausgezeichneter Untersuchungsmethoden bleibt immer noch die Unsicherheit, bei der Feinnadelaspiration nicht genügend oder das falsche $\mathrm{Ma}$ terial gefördert zu haben. Auch die Experten in der vorliegenden Untersuchung waren dagegen nicht ganz gefeit. H. S. FüESSL = 